\title{
Die Einwirkung einiger Gase auf Sulfocyankalium bei höheren Temperaturen.
}

\author{
Von \\ JAROSLAV MHLBAUER. \\ Mit 2 Figuren im Text.
}

In einer früheren Arbeit ${ }^{1}$ wurde darauf hingewiesen, dafs die Temperatur, bei welcher geschmolzenes Sulfocyankalium sich blau färbt, bei $405^{\circ}$ liegt. In der nachfolgenden Studie soll diese Erscheinung noch eingehender behandelt werden.

Schon NöLuNER ${ }^{2}$ hat darauf hingewiesen, dafs Sulfocyankalium beim Schmelzen blau wird und dafs diese Färbung dem Schwefel zuzuschreiben sei, der in einer besonderen Modifikation durch Zersetzung des Schwefelcyanids entsteht.

Nach GILLes ${ }^{3}$ dissoziert Sulfocyankalium in der Wärme und es entstehen Cyankalium und Schwefel, letzterer erscheint unter besonderen Verhältnissen blau. Wird zu schmelzendem Sulfocyankalium ein wenig Schwefel zugesetzt, so wird die blaue Färbung dunkler; bei Zusatz von Kaliumbydroxyd oder Kaliumkarbonat zur Schmelze verschwindet die Färbung alsbald.

Die blaue Schwefelmodjfikation bemerkte zuerst WöHLER bei Reduktion von Eisenoxydsalzen mit Schwefelwasserstoff. VoGkL, SchifF u. a. bestätigten diese Erscheinung.

In neuer Zeit führte OnLow ${ }^{5}$ einige Fälle der Entstehung der blauen Schwefelmodifikation an und nahm als Grund ein Schwefelmolekül $\mathrm{S}_{3}$ analog Ozon $\mathrm{O}_{3}$ an.

\footnotetext{
1 Z. anorg. Chem. 1904, 433.

2 Chem. News 83, 373.

3 Lieb. Ann. 108, 20.

4 Lieb. Ann. 86, 373.

${ }^{5}$ Chem. Centrbl. 1901 II, 522.
} 
Um die Frage zu entscheiden, ob beim Schmelzen von Sulfocyankalium Dissoziation stattfindet, würde im folgenden dieses in verschiedenen indifferenten Gasen bei Rotglut und höher erhitzt, um zu bewirken, dafs der entstehende Schwefel leichter vom Cyankalium getrennt und nachgewiesen werden könnte. Auch durch Erhitzen unter vermindertem Drucke suchte ich diesem Ziele näher zu kommen. Endlich dehnte ich meine Studien auch auf Gase aus, die bei Rotglut auf Sulfocyankalium einwirken.

\section{Erhitzen von Sulfocyankalium unter vermindertem Drucke.}

Die Versuche wurden mit vollikommen reinem, zweimal aus $96 \%$ igen Alkohol umkristallisierten, im Vakuum bei $100^{\circ}$ bis zum konstanten Gewicht getrockneten Sulfocyankalium ausgeführt. Eine bestimmte Menge kam in eine an einem Ende zugeschmolzene Röhre A (Fig. 1) aus schwerschmelzbarem Glase, diese wurde dann

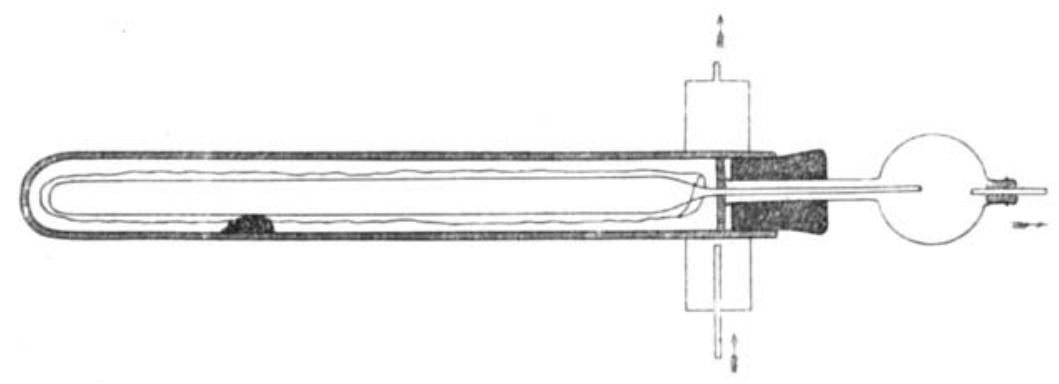

Fig. 1.

am anderen Ende zu einer offenen Spitze ausgezogen, mit Asbestpapier umwickelt und in ein eisernes, einseitig geschlossenes Rohr gesteckt. An das offene Ende des letzteren war ein hohler, zylinderförmiger Metallkühler angelötet, um das Röhrenende abzukühlen und den Kautschukpfropfen zu schützen. Mittelst einer Glasröhre mit kugelförmiger Erweiterung wurde die Verbindung des ganzen Apparates mit einer guten Luftpumpe vermittelt. Die beigefügte Figur 1 zeigt die Einrichtung. Auf diese Weise war es möglich, die Glasröhre sogar während eines halbtägigen Glühens intakt zu erhalten. Zum Glühen diente ein kleiner ErLenmayerscher Ofen. Die Operation wurde einmal bei einem Drucke von $150 \mathrm{~mm}$ während 5 Stunden, das zweite Mal bei einem Drucke von $60 \mathrm{~mm}$ während 4 Stunden durchgeführt. Der nach der Gleichung: $\mathrm{KSCN}=\mathrm{KCN}+\mathrm{S}$ 
entstehende Schwefel sollte am Röhrenende, resp. in der kugelförmigen Erweiterung aufgefangen werden. Eine Ablagerung von Schwefel wurde im Verlaufe der Versuche erst bei Steigerung der Temperatur zur hellen Rotglut beobachtet, also bei einer Temperatur, die weit tiber jener liegt, bei welcher das schmelzende Schwefelcyankalium sich blau färbt. Nach beendeter Erhitzung wurden in beiden Fällen im ausgezogenen Röhrenende unwägbare Mengen eines weifslichen Sublimats und gelber Kügelchen beobachtet, welche Schwefel enthielten und in Schwefelkohlenstoff löslich waren. Der Rückstand enthielt neben unzersetztem Sulfocyankalium Spuren von Sulfid und Cyankalium.

Abgewogen: $1.002 \mathrm{~g}$ KCNS. Im Rückstande

Gefunden: $0.0208 \mathrm{~g} \mathrm{KCN}$

$0.0246 \mathrm{~g} \mathrm{~K}_{2} \mathrm{~S}$ und

$0.8273 \mathrm{~g}$ KCNS; es wurden mithin $7.46 \%$ Sulfocyankalium zerlegt.

Ähnlich war der Vorgang im zweiten Falle.

Verwendet: $2 \mathrm{~g}$ KCNS. Nach dem Erhitzen im Rückstande gefunden $0.0091 \mathrm{~g} \mathrm{KCN}, 0.0105 \mathrm{~g} \mathrm{~K}_{\mathrm{g}} \mathrm{S}$ und $1.960 \mathrm{~g} \mathrm{KCN}$, wurde also $2.0 \% \mathrm{KCNS}$ zerlegt.

Diese Beobachtungen führten zu folgenden Schlufsfolgerungen:

1. Eine vollständige Dissoziation im Vakuum findet unter diesen Umständen nicht statt.

2. Die unbedeutende Menge des so gebildeten Kaliumsulfids und Schwefels kann auf Rechnung einer kleinen, nicht entfernten Sauerstoffmenge gesetzt werden nach der Gleichung:

$$
2 \mathrm{KCNS}+\mathrm{O}_{2}=\mathrm{K}_{2} \mathrm{~S}+\mathrm{S}+2 \mathrm{CO} \text {. }
$$

3. Das gebildete Cyankalium dürfte darauf hindenten, dafs entweder eine geringe Dissoziation stattfand, oder dafs das so gebildete Kohlenoxyd im später auszuführenden Sinne reagiert.

\section{Erhitzung im Stickstoffstrome.}

Reiner Stickstoff wurde nach TishBonN ${ }^{1}$ dargestellt und durch ein Gemisch von konzentrierter Schwefel- und Chromsäure geleitet und im Gasometer gesammelt. Aus diesem wurde das Gas durch alkalische Pyrogallollösung geleitet, weiter mit Chlorcalcium und

1 Chem. News 59, 171. 
konzentrierter Schwefelsäure getrocknet und endlich durch eine kurze, mit glühenden Kupferspiralen gefüllte Röhre geführt.

Die Röhre, in welcher das Sulfocyankalium geglüht wurde, war an einer Stelle etwas ausgezogen; beide Enden waren ein wenig erhöht, so dals auf das Sulfocyankalium das Gas in einer grofsen Fläche einwirken konnte. Die entweichenden Gase wurden durch Wasser und Kalilauge geleitet.

Nach halbstündiger Erhitzung zur hellen Rotglut im Stickstoffstrome wurde gleichfalls im Stickstoffstrome abkühlen gelassen. Aufser der schneeweifsen Schmelze enthielt die Röhre am nicht erhitzten Ende eine unbedeutende Menge ron gelben aus Schwefel bestehenden Tropfen. Die Röhre wurde quantitativ ausgespült und die so erhaltene Lösung analysiert. Dieselbe enthielt Sulfocyankalium nebst Spuren von Kaliumcyanid. Aus $2 \mathrm{~g}$ KCNS resultierten $1.9732 \mathrm{~g}$ KCNS und $0.0184 \mathrm{~g} \mathrm{KCN}$. Es wurden demnach unbedeutende Mengen Schwefelcyankalium $1.35 \%$ im ersten; $2.62 \%$ im zweiten Versuche zersetzt.

Aus diesen Resultaten kann geschlossen werden, dals Stickstoff in der Rotglut auf Sulfocyankalium nicht einwirkt, es fand nur eine unbedeutende Dissoziation gemäfs der Gleichung $\mathrm{KCNS}=\mathrm{KCN}+\mathbf{S}$ statt.

\section{Erhitzen im Kohlenoxydstrome.}

Kohlenoxyd, durch Zersetzung von Ameisensäure durch Schwefelsäure gewonnen, wurde aus einem Gasometer durch Kalilauge und alkalische Pyrogallollösung gewaschen, und mittelst Calciumchlorid und Schwefelsäure getrocknet. Die Röhre wurde vorerst im vorderen Teile, wo das Kohlenoxyd eintrat, hierauf in der Mitte, wo das eingewogene Sulfocyankalium sich befand, erhitzt. Der letzte Teil der Röhre wurde überhaupt nicht erhitzt. Die Röhre wurde mit einem Aspirator mit Wasser, das mit reinem Paraffinöl überschichtet war, verbunden; nachdem die Luft durch Kohlenoxyd verdrängt war, wurde eine halbe Stunde lang zur intensiven Rotglut erhitzt.

$5 \mathrm{~g}$ des untersuchten KCNS ergaben in der Schmelze $4.992 \mathrm{~g}$ KCNS und Spuren von KCN. Das entweichende Gas enthielt eine Schwefelverbindung. Dasselbe wurde mit Sauerstoff gemischt nnd die Mischung durch eine mit erhitztem Platinasbest gefüllte Röhre geleitet; die Verbrennungsprodukte wurden durch eine mit Wasser gefüllte Waschflasche geleitet. Der Inhalt der Waschflasche, als auch das zum Ausspülen der Röhre verwendete Wasser wurde auf Schwefelsåure geprüft. Es wurden $5.2 \mathrm{mg}$ Baryumsulfat erhalten, entsprechend $0.7 \mathrm{mg}$ Schwefel.

7. snorg. Chem. Bd. 49. 
Aus der Anwesenheit einer gasförmigen Schwefelverbindung, wahrscheinlich COS, kann auf eine unbedeutende Dissoziation des Sulfocyankaliums geschlossen werden.

\section{Erhitzung im Wasserstoffstrome.}

Nach J. Playfair ${ }^{1}$ resultiert beim Erhitzen von Sulfocyankulium im Wasserstoffstrome Cyankalium mit einer fast gleichen Menge von Kaliumsulfid.

Conroy, Hesuop und Shorks ${ }^{2}$ teilen mit, dafs Wasserstoff in der Hitze auf Sulfocyankalium nach der Gleichung

$$
\mathrm{KCNS}+2 \mathrm{H}=\mathrm{KCN}+\mathrm{H}_{2} \mathrm{~S}
$$

einwirkt und dafs auf diese Weise ca. 70\% Kaliumcyanid resultieren. Dabei reagiert auch, wie $\mathrm{S}_{\triangle \mathrm{NDM}} \mathrm{TNN}^{3}$ beobachtete, der Schwefelwasserstoff auf Cyankalium:

$$
2 \mathrm{KCN}+\mathrm{H}_{2} \mathrm{~S}=\mathrm{K}_{2} \mathrm{~S}+2 \mathrm{HCN} \text {. }
$$

In den gasförmigen Produkten wurde auch Ammoniak aufgefunden, dessen Bildung durch Verwendung feuchten Sulfocyankaliums verursacht wurde.

Zu den folgenden Versuchen wurde trockenes Sulfocyankalium verwendet. Das Wasserstoffgas wurde durch Chromschwefelsäure, hierauf durch alkalische Kupferlösung gereinigt, dann über glühende Kupferspiralen geleitet und endlich durch Calciumchlorid und hierauf durch Schwefelsäure getrocknet. Später wurde das Gas durch Alkalilauge, konzentrierte Schwefelsäure und Überleiten über metallisches Natrium, das sich in einer kurzen, schwach bis zur Hydridbildung erhitzten Röhre befand, gereinigt. Es wurde bei lichter Rotglut über eine Schicht von Sulfocyankalium während 3 Stunden geleitet. Die flüchtigen Produkte wurden in konzentrierter Natronlauge aufgefangen. Nach stattgefundener Abkühlung im Wasserstoffstrome wurde der lichtrote Rückstand analysiert und die in der Lauge absorbierten Produkte bestimmt. Der Ruckstand war ein Gemisch von Cyankalium und Kaliumsulfid. Unter den flüchtigen Produkten wurden Schwefel- und Cyanwasserstoff, aber kein Ammoniak nachgewiesen.

1 J. Soc. Chem. Ind. 11, 16.

S. Soc. Chem. Ind. 20, 320-322; vergl. Ohem. Centrbl. 1901 I, 1278.

3. Soc. Chem. Ind. 20, 820; vergl. Chem. Centrbl. 1902 II, 18. 
Verwendet wurden $1.3824 \mathrm{~g}$ KCNS.

Im Rückstande waren $0.582 \mathrm{~g} \mathbf{K}_{2} S$ und $0.455 \mathrm{~g}$ KCN.

An gasförmigen Produkten wurde bestimmt:

$0.3675 \mathrm{~g} \mathrm{H}_{2} \mathrm{~S}$ und $0.1951 \mathrm{~g} \mathrm{HCN}$.

Ein ähnliches Resultat ergab. ein zweiter Versuch:

Verwendet $1.501 \mathrm{~g}$ KCNS; im Rückstande gefunden $0.5026 \mathrm{~g}$ KCN und $0.4524 \mathrm{~g} \mathrm{~K} \mathrm{~K}_{\mathrm{p}} \mathrm{S}$; in dem gasförmigen Produkten: $0.3961 \mathrm{~g} \mathrm{H}_{2} \mathrm{~S}$ und $0.2181 \mathrm{~g}$ HCN.

In den Schmelzen waren Cyankalium und Kaliumsulfid im Molekularverhältnis 2:1 enthalten, in den gasförmigen Produkten ist das Verbältnis von Schwefelwasserstoff zum Cyanwasserstoff 3:2, woraus die Reaktionsgleichung sich ergibt:

$$
4 \mathrm{KCNS}+8 \mathrm{H}=2 \mathrm{KCN}+\mathrm{K}_{2} \mathrm{~S}+3 \mathrm{H}_{2} \mathrm{~S}+2 \mathrm{HCN} \text {. }
$$

Von Interesse ist, dals nach A. W. Hofrmass bei Einwirkung von Wasserstoff in statu nascendi auf Rhodanwasserstoffsäure ähnliche Produkte entstehen

$$
\underset{\mathrm{CN}}{\mathrm{H}}>\mathrm{S}+\underset{\mathrm{H}}{\mathrm{H}}=\mathrm{HCN}+\mathrm{H}_{\mathbf{2}} \mathrm{S} \text {. }
$$

Einwirkung von Wasserdampf anf Sulfocjankalinm bei höherer

$$
\text { Temperatar. }
$$

Vorläufige Versuche in Röhren von schwerschmelzbarem Glase, die jedoch durch den über $400^{\circ}$ erhitzten Wasserdampf durchwegs zersprangen, ergaben, dafs Sulfocyankalium durch Wasserdampf völlig zersetzt wird, und flüchtige Produkte resultieren, unter denen Kohlendioxyd, Schwefelwasserstoff und Ammoniak konstatiert werden konnten. Der nichtflüchtige Rückstand enthielt Kaliumsulfid und Kaliumkarbonat. Während der Arbeit konnte beobachtet werden, wie die Schmelze eine Weile sich blau färbt, nach und nach dunkler und endlich dunkelbraun wurde. Nach der Abkühlung ist die Reaktionsmasse rot.

Für die weiteren Versuche wurde ein eigens dazu konstruierter Apparat verwendet.

In einem Autoklav wurde Wasser unter einem Druck von zwei Atmosphären zum Kochen erbitzt, der Dampf wurde durch den kupfernen, zur Rotglut erhitzten Vorwärmer (a) geleitet; dann durch die gebogene Porzellanröhre $(b)^{1}$ auf einen kleinen Porzellantiegel $(c)$,

1 Die Verbindung des Metallrohres (a) mit dem Porzellanrohre (b) wurde durch eine über die zusammenstofsenden Enden geschobene anliegende Messinghülse und die Abdichtung durch umwickeltes mit Wasser benetztes Asbestpapier erzielt. 
der in einem gröfseren Tontiegel auf einer Schicht ausgeglühten reinen Sandes stand. Der Porzellantiegel war mit einer durchbohrten Glimmerscheibe $(d)$ bedeckt, der grofse Tontiegel mit einer grölseren (e), die in den Tiegel mit Gips derart gedichtet war, dafs eine unbedeckte runde Öffnung zur Beobachtung der stattfindenden Reaktion ubrig blieb. Endlich war der Tontiegel mit dem Hauptdeckel $(f)$ versehen. Gegenüber der Eintrittsöffnung des Dampfrohres befand sich eine Öffinung zum Einsatz der Porzellanröhre $(g)$.

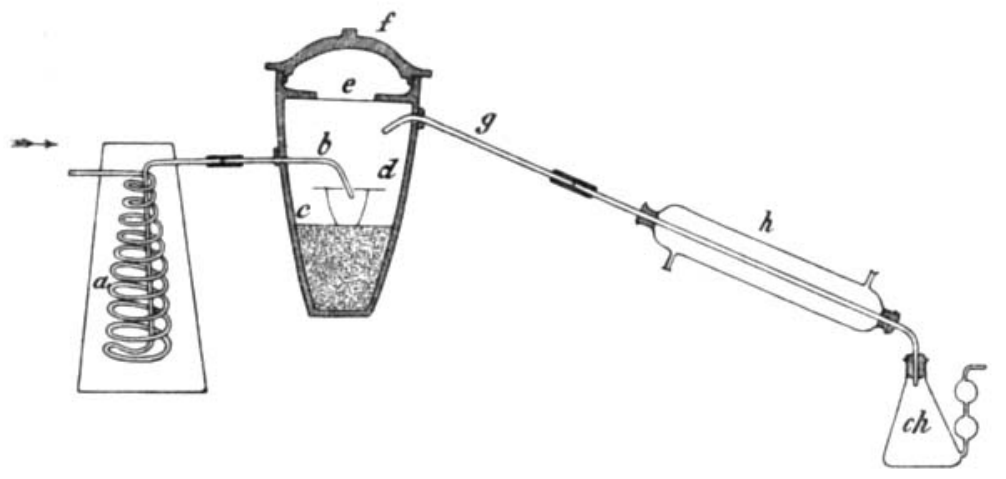

Fig. 2.

Die Dichtung der beiden Porzellanröhren in der Tiegelwand geschah durch Gips. Die flüchtigen Produkte gelangten durch einen kurzen LIEBIG schen Kühler $(h)$ in Absorptionsgefäfse, von denen nur das erste VoLHARDsche in der Abbildung gezeichnet ist.

Beim ersten Versuch wurden in den Porzellantiegel $3.4166 \mathrm{~g}$ Sulfocyankalium abgewogen, der ganze Apparat zusammengestellt und alle Teile, durch welche der Dampf geleitet werden sollte, erwärmt; der Tontiegel wurde durch einen Revolverbrenner erhitzt. Sobald das geschmolzene Sulfocyanid die blaue Färbung annahm, wurde der stark überbitzte Dampf eingelassen. Während der erfolgten Zersetzung entwickelten sich Gase und die Schmelze färbte sich fast schwarz; die Erhitzung dauerte etwa 40 Minuten.

Die Reaktionsmasse enthielt $0.9681 \mathrm{~g} \mathrm{~K}_{2} \mathrm{~S}$ und $1.2155 \mathrm{~g} \mathrm{~K}_{2} \mathrm{CO}_{2}$. Das Molekularverhältnis dieser so gebildeten Stoffe war 1:1.

Im zweiten Falle wurden $7.5502 \mathrm{~g}$ KCNS verwendet und die flüchtigen Produkte in konzentrierter Kalilauge und wässeriger Schwefelsäure aufgefangen. Der feste Rückstand enthielt $2.6801 \mathrm{~g}$ $\mathrm{K}_{2} \mathrm{CO}_{3}$ und $2.132 \mathrm{~g} \mathrm{~K}_{2} \mathrm{~S}$. Im Destillat wurden gefunden: $2.5605 \mathrm{~g}$ $\mathrm{CO}_{2}, 1.9766 \mathrm{H}_{2} \mathrm{~S}$ und $1.3107 \mathrm{~g} \mathrm{NH}_{3}$. 
Das Verhältnis zwischen $\mathrm{K}_{2} \mathrm{~S}$ und $\mathrm{K}_{2} \mathrm{CO}_{3}$ erwies sich wie im ersten Falle, im Destillat näherte sich das Molekularverhältnis $\mathrm{NH}_{3}: \mathrm{CO}_{2}: \mathrm{H}_{2} \mathrm{~S}=4: 3: 3$.

Hieraus folgt nachfolgender Verlauf der Reaktion:

$$
4 \mathrm{KCNS}+9 \mathrm{H}_{2} \mathrm{O}=\mathrm{K}_{2} \mathrm{CO}_{3}+\mathrm{K}_{2} \mathrm{~S}+3 \mathrm{CO}_{2}+3 \mathrm{H}_{2} \mathrm{~S}+4 \mathrm{NH}_{3} \text {. }
$$

Diese Versuche erklären die schon früher bemerkte Zersetzung von feuchtem ${ }^{1}$ Sulfocyankalium und die Bildnng von alkalischem Sulfide bei der Schmelzung.

Sodann wurde eine Reihe weiterer Versuche über den Einflufs von Wasser unter Druck auf Sulfocyankalium ausgeführt. Einerseits wurde versucht, festzustellen, wie sich $1 / 10$ n. KCNS-Lösung unter Druck verhält, andererseits wurde eine gröfsere Menge von Sulfocyankalium mit einigen Tropfen Wasser in einer geschlossenen Röhre im Bombenofen erhitzt. Eine $1 / 10$ n. Sulfocyankaliumlösung wurde beim fünfstündigen Erhitzen in einem geschlossenen Glasrohr auf $170^{\circ}-180^{\circ} \mathrm{C}$ nicht im geringsten zersetzt, ebenso auch zeigte eine Lösung, deren $1 \mathrm{ccm} 24.8 \mathrm{mg}$ jener Verbindung enthielt auf $180-190^{\circ} \mathrm{C}$ nach fünfstündigem Erhitzen keinerlei Zersetzung.

Die Versuche, die Erhitzung über $200^{\circ}$ zu steigern, mifslangen, da jedesmal die Glasröhren während des Versuches zertrümmert wurden.

Beim Erhitzen in einem geschlossenen Rohre von $2 \mathrm{~g} 100 \%$ igen Sulfocyankalium mit $1 / 2 \mathrm{ccm}$ Wasser sechs Stunden auf $200^{\circ}$ wurde nach erfolgter Abkühlung beim Öffnen der Röhre ein unbedeutender Druck bemerkt. Gefunden waren Sulfid, Ammoniak neben überschüssigem Sulfocyankalium.

Es wurden gefunden: $0.0182 \mathrm{~g} \quad \mathrm{~K}_{2} \mathrm{~S}, 0.0095 \mathrm{~g}\left(\mathrm{NH}_{4}\right)_{2} \mathrm{~S}$ und $1.9733 \mathrm{~g}$ KCNS.

Die Zersetzung erlitt nur eine unbedeutende Menge (1,34\%) des Sulfocyankaliums und dies wahrscheinlich nach der Gleichung

$$
2 \mathrm{KCNS}+4 \mathrm{H}_{2} \mathrm{O}=\mathrm{K}_{2} \mathrm{~S}+\left(\mathrm{NH}_{4}\right)_{2} \mathrm{~S}+2 \mathrm{CO}_{2} \text {. }
$$

\section{Einwirkung von Schwefelwasserstoff auf Sulfocyankalium.}

Schwefelwasserstoff wurde aus Antimonglanz mittelst Salzsäure hergestellt. Das sich entwickelnde Gas wurde durch einen Kühler,

I Z. anorg. Chem. 42 (1904), 434. 
durch eine mit Schwefelnatrium beschickte Waschflasche, hierauf durch eine lange, mit Chlorcalcium gefüllte U-Röhre und endlich durch eine in einen Verbrennungsofen gelegte Röhre aus schwerschmelzbarem Glase geleitet. Die flüchtigen Reaktionsprodukte wurden in konzentrierter Natronlauge aufgefangen. Erhitzt wurde nur die Stelle, wo sich das Sulfocyankalium befand und, der Teil der Röhre vor demselben. Der Rückstand nach dem Erhitzen war in der Hitze dunkelbraun, nach dem Erkalten rot; in dem kühlen Röhrenteil war ein gelber Anflug ron Schwefel zu beobachten, in den Waschflaschen wurde Cyanwasserstoff aufgefunden.

In einem Falle wurden: $1.7275 \mathrm{~g}$ KCNS verwendet; die Erhitzung dauerte bei heller Rotglut fast 4 Stunden. Der Rückstand erhielt $0.9706 \mathrm{~g} \mathrm{~K}_{\mathbf{g}} \mathrm{S}$ und Spuren von KCNS. In den Waschflaschen waren $0.4722 \mathrm{~g} \mathrm{HCN}$.

Ein zweites Mal wurden $0.2159 \mathrm{~g}$ KCNS verwendet; die Erhitzung dauerte in Rotglut etwa 1 Stunde. Der Rest enthielt $0.081 \mathrm{~g} \mathrm{~K}_{\mathrm{g}} \mathrm{S}$ und $0.0719 \mathrm{~g}$ KCNS In der Absorptionstlüssigkeit wurde $0.0417 \mathrm{~g}$ HCN gefunden.

Das im Reste gefundene Kaliumsulfid und der im Destillat enthaltene Cyanwasserstoff waren im Molekularverhältnis $1: 2$.

Die Reaktion verläuft mithin folgendermalsen:

$$
2 \mathrm{KCNS}+\mathrm{H}_{2} \mathrm{~S}=\mathrm{K}_{2} \mathrm{~S}+\mathrm{HCN}+2 \mathrm{~S} \text {. }
$$

Es ist jedoch auch möglich, dafs die Reaktion in zwei Phasen verläuft: nämlich

$$
\begin{gathered}
\text { 1. } \mathrm{KCNS}=\mathrm{KCN}+\mathrm{S} . \\
\text { 2. } 2 \mathrm{KCN}+\mathrm{H}_{2} \mathrm{~S}=\mathrm{K}_{2} \mathrm{~S}+2 \mathrm{HCN}^{1}
\end{gathered}
$$

Schmelzung von Sulfocyankalium im Kohlendioxydstrome.

Eine abgewogene Menge von Sulfocyankalium würde im Porzellanschiffchen zu gelinder Rotglut im trockenen Kohlendioxydstrome erhitzt.

Nach $1 / 2$ stündiger Erhitzung war die Schmelze nach dem Abkühlen reinweifs und enthielt neben überschüssigem Sulfocyankalium Kaliumcyanat; im kalten Ende des Rohres war eine geringe Menge gelber Schwefeltropfen.

Verwendet: $1.1575 \mathrm{~g} \mathrm{KCNS}$; in dem Rückstande wurden gefunden $1.0916 \mathrm{~g}$ KCNS und $0.0405 \mathrm{~g}$ KCNO; der Zersetzung verfielen mithin $5.7 \% \mathrm{KCNS}$.

In einem zweiten Falle resultierte eine Schmelze mit $96.53 \%$ KCNS und $3.40 \%$ KCNO.

1 Sanduand, J. Soc. Chem. Ind. 20, 120; vergl. Chem. Centrbl. 1900 II, 18. 
Das Kaliumcyanat konnte auf zweierlei Weise entstehen:

a) entweder durch teilweise Dissoziation, wobei das Cyankalium mit Kohlendioxyd unter Bildung von Kaliumcyanat und Kohlenoxyd reagierte: ${ }^{1}$

$$
\mathrm{KCNS}=\mathrm{KCN}+\mathrm{S} ; \mathrm{KCN}+\mathrm{CO}_{2}=\mathrm{KCNO}+\mathrm{CO} ;
$$

b) oder es fand blofs die folgende Reaktion statt:

$$
\begin{gathered}
\mathrm{KCNS}+\mathrm{CO}_{2}=\mathrm{KCNO}+\mathrm{COS} . \\
\mathrm{COS} \rightleftarrows \mathrm{CO}+\mathrm{s} .
\end{gathered}
$$

In den nachfolgenden Versuchen wurden auch die flüchtigen Produkte berücksichtigt.

$0.7171 \mathrm{~g}$ KCNS wurden 1 Stunde hindurch im Kohlendioxydstrome erhitzt, das Gas aufgefangen und nach Entfernung des überschüssigen Kohlendioxyds das gebildete Kohlendioxyd mit saurer Kupferchlorürlösung absorbiert.

Die Schmelze enthielt: $0.6182 \mathrm{~g}$ KCNS und $0.0685 \mathrm{~g}$ KCNO. Absorbiert wurden $19 \mathrm{cem}$ Kohlenoxyd bei $22.5^{\circ}$ und $748 \mathrm{~mm}$ Barometerstand, was einem Quantum von $20.9 \mathrm{mg}$ CO entspricht.

Die Molekularmengen des resultierenden Kaliumcyanats und Kohlenoxyds sind annähernd proportional. In einem analog ausgeführten Versuch wurde nur die Stelle, wo das Sulfocyankalium sich befand, erhitzt, und dies aus dem Grunde, um die Zersetzung des Karbonylsulfids in Kohlenoxyd und Schwefel zu verhüten. Die Erhitzung in heller Rotglut dauerte etwa 2 Stunden.

Verwendet: $0.4729 \mathrm{~g}$ KCNS. Das Karbonylsulfid wurde nach Entfernung des überschüssigen Kohlendioxyds im Kruson schen Absorptionsmittel ${ }^{2}$ absorbiert und wurden $100.7 \mathrm{ccm} \operatorname{COS}$ beim Barometerstande von $748 \mathrm{~mm}$ und $22.5^{\circ}$ gefunden, entsprechend $0.2393 \mathrm{~g}$ COS.

\section{Die Reaktionsschmelze enthielt: $0.0295 \mathrm{~g}$ Schwefel das erhaltene COS enthielt: $0.1276 \mathrm{~g}$ Schwefel}

Im ganzen 0.1571 g Schwefel

Das in Verwendung gekommene

$$
\frac{\text { Sulfocyankalium enthielt: } 0.1559 \mathrm{~g} \text { Schwefel }}{\text { Differenz: } 0.0012 \mathrm{~g} \text { Schwefel }}
$$

\section{Einwirkung von Schwefeldioxyd anf Snlfocyankalium.}

Die Versuche wurden wie bei Anwendung von Kohlendioxyd ausgeführt. Die Erhitzung fand bei heller Rotglut statt. Die

1 Eiroant, Chem. Centrbl. 1886, 770.

2 Zeitschr. analyt. Chem. 1882, 79. 
flüchtigen Produkte wurden in eine VoLHardsche Waschflasche geleitet.

Beim ersten Versuche wurden 0.3958 g KCNS verwendet. Die Erhitzung dauerte 1 Stunde.

Das Sulfocyankalium schmolz kurze Zeit hindurch mit blauer Farbe, färbt sich hierauf dunkelbraun und wurde endlich wieder hell. Die Reaktionsmasse wurde im Schwefeldioxydstrome erkalten gelassen. Dieselbe ist reinweifs. Am kühlen Ende des Glasrohres war ein mächtiger Anflug von Schwefel. Das Reaktionsprodukt ist reines Kaliumsulfat. Gewonnen wurden $0.3528 \mathrm{~g} \mathrm{~K}_{2} \mathrm{SO}_{4}$.

Bei einem weiteren Versuche wurden $10 \mathrm{~g}$ KCNS verwendet und nur kürzere Zeit (etwa eine $1 / 2$ Stunde) gegluht, so dafs der Rückstand braungefärbt blieb; derselbe enthielt Kaliumsulfid und geringere Mengen an Kaliumsulfat und Sulfocyankalium.

Hieraus folgt, dafs die Reaktion in wenigstens zwei Stufen verläuft. In der ersten entsteht Kaliumsulfid neben Schwefel, in der zweiten reagiert das Kaliumsulfid mit dem Schwefeldioxyd nach den Gleichungen:

$$
\begin{gathered}
2 \mathrm{KCNS}+2 \mathrm{SO}_{2}=\mathrm{K}_{2} \mathrm{~S}+2 \mathrm{CO}_{2}+2 \mathrm{~N}+3 \mathrm{~S} \\
\mathrm{~K}_{2} \mathrm{~S}+2 \mathrm{SO}_{2}=\mathrm{K}_{2} \mathrm{SO}_{4}+2 \mathrm{~S} .
\end{gathered}
$$

Hiernach mufs es möglich sein, durch Bestimmung des freien Stickstoffes das Mals der Zersetzung nach der ersten Gleichung zu bestimmen. Die zweite Gleichung ist direkt zu beweisen.

$4.03 \mathrm{~g}$ Sulfocyankalium wurden eine $1 / 2$ Stunde im Schwefeldioxydstrome geglüht und die gebildeten Gase in einem mit konzentrierter Salzlösung gefüllten Gasometer gesammelt. Die Menge des Gases betrug $794.5 \mathrm{ccm}$ bei $750 \mathrm{~mm}$ Druck und $22^{\circ}$. Aus $50 \mathrm{ccm}$ wurde vorerst mittels Kalilauge, Schwefeldioxyd und Koblendioxyd entfernt, und der Rest mit salzsaurem Kuprochlorid behandelt; es erfolgte jedoch keine Absorption. Der Rest von $28.8 \mathrm{ccm}$ bestand aus Stickstoff. Durch Umrechnung anf die ursprüngliche Gasmenge resultierten $492.8 \mathrm{ccm}$. Im festen Rückstande war nur eine kleine Menge von unzersetztem KCNS, dessen Stickstoffmenge nach der KJELdaru schen Methode bestimmt wurde.

Die ursprüngliche Sulfocyankaliummenge enthielt: $0.577 \mathrm{~g} \mathrm{~N}$.

$$
\begin{array}{lll}
\text { Im Gasform gefunden: } & 0.553 \mathrm{~g} \mathrm{~N}\} & 0.5784 \mathrm{~g} \mathrm{~N} \\
\text { Im unzersetzten KCNS: } & \frac{0.0254 \mathrm{~g} \mathrm{~N}}{\text { Differenz : }}+\mathbf{+} 0.0084 \mathrm{~g} \mathrm{~N} .
\end{array}
$$

Um auch die zweite Stufe der Reaktion zu beweisen, wurde eine abgewogene Menge wasserfreien Natriumsulfids im Porzellanschiffchen in einem raschen Schwefeldioxydstrome zum Rotglühen erhitzt. Nach $1 \frac{1}{2}$ stündigem Erhitzen und Abkühlung im Schwefel- 
dioxydstrome resultierte als Rückstand eine weifse Masse und als Sublimat Schwefel.

$$
\begin{aligned}
\text { Verwendet wurden: } & 0.4358 \mathrm{~g} \mathrm{Na}_{2} \mathrm{~S} \\
\text { Die Schmelze enthielt: } & 0.4470 \mathrm{~g} \mathrm{SO}_{3} \\
& 0.3465 \mathrm{~g} \mathrm{Na}_{2} \mathrm{O}
\end{aligned}
$$

welches Verhältnis der Zusammensetzung von Natriumsulfat entepricht.

Die Reaktion verlief mithin in der oben angedeuteten Weise (2. Stufe).

In der Literatur findet sich nur die umgekehrte Reaktion angeführt: Erhitzt man nämlich das Sulfat mit überschüssigem Schwefel, entweicht $\mathrm{SO}_{2}$ und bildet sich Sulfid.

SEsTINI ${ }^{1}$ beobachtete diese Reaktion beim Erhitzen von Calciumsulfat mit Schwefel bei $450^{\circ}$ und VronI ${ }^{2}$ verwies darauf, dafs die Reaktion allgemeinerer Natur sei; es gelte die schematische Gleichung:

$$
\mathrm{RSO}_{4}+\mathrm{S}_{2}=\mathrm{RS}+2 \mathrm{SO}_{2} \text {, }
$$

und zwar für die Sulfate der Metalle $\mathrm{Al}, \mathrm{Cr}, \mathrm{Zn}, \mathrm{Mn}, \mathrm{Fe}, \mathrm{Cd}, \mathrm{Pb}$, $\mathrm{Bi}, \mathrm{Cu}, \mathrm{Sb}$ und $\mathrm{Hg}$.

Doch hatte schon vorher STOLBA ${ }^{3}$ gefunden, dafs durch Schmelzung von wasserfreiem Eisensulfat mit Schwefel reines Eisensulfid dargestellt werden kann.

Es gilt demnach die Gleichung:

$$
\mathrm{K}_{2} \mathrm{~S}+2 \mathrm{SO}_{2} \rightleftarrows \mathrm{K}_{2} \mathrm{SO}_{4}+2 \mathrm{~S}
$$

je nachdem Schwefel oder Schwefeldioxyd im Überschusse vorhanden ist.

Durch diese umkehrbare Reaktion finden die Unuschen ${ }^{4}$ Versuche über die Einwirkung von Schwefeldioxyd auf Metalle bei Rotglut eine Erklärung. Es entstanden Sulfide, eventuell aber auch Sulfate. Die Reaktion dürfte wohl auch hier in zwei Phasen verlaufen, vorerst bildet sich das Sulfid und hierauf entstand aus dem Sulfid durch überschüssiges Schwefeldioxyd das betreffende Sulfat.

1 Bull. soc. chim. Paris (N. S.) 24. 490.

2 Ber. deutsch. chem. Ges. 10a, 293; Chem. Centrbl. 1877, 273.

3 Journ. prakt. Ohem. 99, 54.

4 Ber. deutsch. chem. Ges. 23 II, 2151.

Prag, Chem. Laboratorium der k. k. böhm. techn. Hochschule.

Bei der Redaktion eingegangen am 9. Februar 1906. 